Revista General de Información y Documentación ISSN: 1132-1873

http://dx.doi.org/10.5209/RGID.60815

\title{
Estrategias y recursos de difusión y promoción digital de la investigación en el sistema universitario español. Análisis de las universidades públicas a través de su web institucional
}

\author{
Brenda Siso Calvo ${ }^{1}$; Rosario Arquero Avilés ${ }^{2}$; Gonzalo Marco Cuenca ${ }^{3}$
}

Recibido: 9 de enero 2018 / Aceptado: 10 de mayo de 2018

Resumen. Esta investigación tiene como objetivo principal realizar un análisis comparativo sobre el estado actual en materia de difusión y promoción digital de la investigación en el sistema universitario español. Para cumplir con dicho objetivo se examina la difusión y promoción que hacen las universidades públicas, a partir de sus páginas web institucionales, de la información de la actividad investigadora y qué recursos utilizan para permitir el acceso a dicha información, así como conocer qué estrategias prevén desarrollar en el futuro, mediante el estudio de las tendencias y líneas de actuación en este contexto contempladas en sus planes y/o documentos estratégicos.

Para llevar a cabo el estudio, se han seleccionado y definido previamente una serie de indicadores para, seguidamente, realizar una revisión y recogida sistemática y normalizada de datos por medio de la exploración de las páginas web de las 50 universidades públicas incluidas en el Registro de Universidades, Centros y Títulos (RUCT).

Los principales resultados obtenidos permiten diagnosticar que se perciben avances y considerables progresos, aunque existe un amplio margen de mejora para alcanzar el nivel de implantación deseable en lo que a existencia de recursos específicamente orientados a la difusión y promoción digital de la investigación se refiere, identificándose también diversas iniciativas que permiten determinar el creciente interés de las instituciones analizadas por reforzar sus estrategias en esta línea.

Palabras clave: Difusión digital de la investigación; Acceso a la información; Marketing científico; Marketing digital; Reputación institucional; Marca institucional; Visibilidad científica; Promoción de la actividad científica e investigadora; Sistema universitario español; Páginas Web Universitarias.

\section{[en] Strategies for the Digital Dissemination and Promotion of Research in the Spanish University System. Analysis of Public Universities through their Institutional Websites}

\footnotetext{
Abstract. The main objective of this research focuses on a comparative analysis of the current state of digital dissemination and promotion of research in the Spanish University System. For achieve the objective this study focuses on examine the dissemination and promotion of research activities made by public universities, and what resources they use to allow access to this information by means of

1 Universidad Complutense de Madrid. Departamento de Biblioteconomía y Documentación.

E-mail: msiso@ucm.es

2 Universidad Complutense de Madrid. Departamento de Biblioteconomía y Documentación.

E-mail: carquero@ucm.es

3 Universidad de Zaragoza. Departamento de Ciencias de la Documentación e Historia de la Ciencia.

E-mail: gmarco@ucm.es
} 
their institutional websites. The paper also identifies what strategies universities plan to develop in the nearest future by studying the trends and lines of action contemplated in their plans and/or strategic documents.

A systematic and standardized review and data collection was used as methodological tool, by exploring the websites of the 50 public universities included in the Registro de Universidades, Centros y Títulos (RUCT), with a series of indicators previously defined.

The results revealed that there has been considerable progress, although there is still a long way to go before the desired level of implementation in terms of availability of resources specifically aimed at the digital dissemination and visibility of research. Lastly, many initiatives have also been identified to determine the growing interest of the institutions in strengthening their strategies along these lines.

Keywords: Digital Dissemination of Research; Information Access; Scientific Marketing; Digital Marketing; Reputation; Institutional Branding; Scientific Visibility; Promotion of Scientific and Research Activity; Spanish University System; University Websites.

Sumario. 1. Introducción, 2. Objetivos y metodología, 3. Resultados y discusión, 4. Conclusiones, 5. Referencias Bibliográficas.

Cómo citar: Siso Calvo, B., Arquero Avilés, R., Marco Cuenca, G. (2018) Estrategias y recursos de difusión y promoción digital de la investigación en el sistema universitario español. Análisis de las universidades públicas a través de su web institucional, en Revista General de Información y Documentación 28 (1), 95-117.

\section{Introducción}

La investigación y la generación de conocimiento se ha consolidado en los últimos años como una actividad fundamental para el progreso y generación de riqueza de la sociedad española. La Ley 14/2011, de 1 de junio, que regula el actual contexto del Sistema Español de Ciencia, Tecnología e Innovación reconoce a las universidades, junto con los Organismos Públicos de Investigación, como los principales pilares responsables de la mayor parte de la actividad investigadora (España, 2011: 54393). En este sentido, la Ley Orgánica 6/2001, de 21 de diciembre, de Universidades (LOU) pone también de manifiesto la importancia de la investigación como factor competitivo para las universidades y, concretamente en su artículo 39.1, destaca que "la investigación, fundamento de la docencia, medio para el progreso de la comunidad y soporte para la transferencia social del conocimiento, constituye una función esencial de las Universidades” (España, 2001: 49410).

La LOU profundiza en los aspectos relacionados con el desempeño de la investigación en el ámbito universitario en sus diversos artículos y dedica expresamente su Título VII a la investigación y transferencia de conocimiento. En su artículo 1c, se subraya como una de las principales funciones de la Universidad "la difusión, la valorización y la transferencia del conocimiento al servicio de la cultura, de la calidad de la vida, y del desarrollo económico” (España, 2001: 49403). Otro de los aspectos contemplados en la LOU, en sus artículos 40.1 y 40.2, es la consideración de la misma como un derecho y un deber del profesorado universitario que, independientemente de la investigación individual, se llevará a cabo principalmente por grupos de investigación e Institutos Universitarios de 
Investigación (España, 2001: 49410). Consecuentemente, las universidades, en el cumplimiento de la función atribuida por el marco normativo de referencia, disponen en su estructura de grupos de investigación, definidos como la entidad fundamental para la generación de conocimiento científico. Por su parte, en la ya mencionada ley 14/2011, también podemos encontrar referencias al fomento de la investigación científica, a la transferencia y transmisión del conocimiento, así como a la difusión y divulgación de los resultados de investigación y, cabe señalar en este aspecto, su artículo 37, dedicado a la difusión en acceso abierto (España, 2011). ${ }^{4}$

Resulta también de interés citar el informe "La Universidad Española en Cifras 2015/2016" donde se facilita información sobre la organización de las estructuras productivas de $\mathrm{I}+\mathrm{D}+\mathrm{i}$, indicando la existencia de un total de 9.335 grupos de investigación, con una media de 194 grupos de investigación por universidad y 8,6 profesores/investigadores por cada grupo (CRUE, 2017).

Adicionalmente y, en línea con lo expuesto, los requisitos normativos, el avance de las iniciativas sobre transparencia del conocimiento y acceso a la información en las instituciones públicas, así como el creciente interés por ajustarse a los criterios de calidad definidos por diversas entidades para elaborar clasificaciones o rankings universitarios, cada vez más difíciles de ignorar por las universidades (Rauhvargers, 2013), ha hecho que la difusión de la información sobre la investigación se haya convertido en uno de los aspectos nucleares en los planes estratégicos de las universidades. En este contexto, la difusión de la información relacionada y generada por las universidades se convierte en la función principal de la web institucional (Pérez-Montoro, 2014)

Además, hay que tener en cuenta que Internet ha revolucionado el modelo tradicional de comunicación científica, proporcionando nuevos canales de comunicación y difusión en un contexto digital, que vienen a facilitar la publicación, el acceso y la promoción de la investigación en el ámbito académico. Tal es así, que en los últimos años han surgido muchas iniciativas relacionadas con la divulgación de la ciencia desde las páginas webs de las universidades, fomentadas en gran parte por las amplias posibilidades que ofrece el propio medio, con formatos más atractivos y multimedia, con una considerable mejora en el acceso e, incluso, con unas ventajas, en términos económicos, mucho más rentables. A todo ello, hay que añadir otro grupo de motivos que llevan a utilizar este canal de una forma estratégica como, por ejemplo, las necesidades de rendición de cuentas de los propios grupos de investigación, la mejora en cuanto a la transparencia de los resultados científicos y, por supuesto, el impulso que se le aporta a la imagen y la calidad de la propia institución como entidad investigadora. Sin embargo, tal y como indican en su estudio Saraite-Sariane, Galvez Rodríguez y Haro de Rosario (2018: 63) cabe señalar que, aunque las universidades son conscientes de la importancia de tener una web institucional navegable, accesible y

4 Concretamente, el artículo 37.1 de la Ley 14/2011 indica “los agentes públicos del Sistema Español de Ciencia, Tecnología e Innovación impulsarán el desarrollo de repositorios, propios o compartidos, de acceso abierto a las publicaciones de su personal de investigación y establecerán sistemas que permitan conectarlos con iniciativas similares de ámbito nacional e internacional” (España, 2011; 54425). 
con buen diseño, la información electrónica es aún la dimensión menos valorada debido a que las universidades centran más su atención en la divulgación de información general que en cuestiones más específicas, como sería el caso de la información relativa al ámbito específico de la investigación.

En cualquier caso, la página web se convierte en la carta de presentación y en el escaparate que los grupos de investigación y las propias universidades utilizan para comunicar y transmitir decisiones institucionales y estratégicas. Para Pinto Molina y otros (2004: 346) la presencia en la web se sitúa “como uno de los pilares estratégicos de los procesos de comunicación internos y externos que sustentan la vida universitaria". Las universidades, por lo general, se dotan de distintas estructuras (portales y repositorios institucionales, oficinas de apoyo a la investigación, etc.) para intentar incrementar la visibilidad de la actividad científica y de los resultados de investigación y, por otro lado, desde la perspectiva del diseño, orientan sus sitios web en relación con los tipos de usuarios que conforman la comunidad universitaria y según las principales funciones de las universidades: docencia, extensión universitaria e investigación (Pérez-Montoro, 2014).

No obstante, divulgación y difusión, conceptos propios del proceso comunicativo de la ciencia, tienen unas características diferenciadoras. La principal característica de la divulgación es que tiene como público objetivo la sociedad en general $\mathrm{y}$, por tanto, la información transmitida tiene que ser sencilla y comprensible, de ahí que sea necesario adaptar el lenguaje científico a un lenguaje común y popular, y que los principales canales de divulgación sean los medios de comunicación de masas (formatos convencionales) e Internet (Seguí Simarro; Poza Luján; Mulet Salort, 2015). La difusión científica hace referencia a la comunicación entre especialistas (Espinosa Santos, 2010), científicos e investigadores con un rigor y una formación que les permite comprender conceptos y textos científicos (Seguí Simarro; Poza Luján; Mulet Salort, 2015). Otra de las características de la difusión es que constituye la etapa final del trabajo efectuado, por lo que se configura como el medio por el cual se dan a conocer los resultados de las investigaciones (Cegarra Sánchez, 2012). Este medio, complementado por los canales digitales, da lugar a la difusión adjetivada como digital, que potencia y facilita la comunicación y el acceso a la información a las partes interesadas.

Si bien el Sistema Universitario Español se enfrenta desde hace varios años a múltiples retos como resultado de diversos factores normativos, sociales y económicos, entre los que cabe resaltar la adaptación al Espacio Europeo de Educación Superior, la globalización y la mayor demanda de conocimiento por parte de la sociedad (Fundación Telefónica, 2012), no cabe duda de que el desarrollo e impacto de las Tecnologías de la Información y la Comunicación (TIC) así como la consolidación de la Sociedad Digital han influido de forma decisiva en la transformación y modernización de las universidades. Una de las cuestiones fundamentales que planteaba la estrategia nacional de modernización de las universidades, Estrategia Universidad $2015^{5}$, hacía referencia a la necesidad de desarrollo de una estrategia de comunicación para transmitir a la sociedad la

\footnotetext{
5 Desarrollada por la Secretaría General de Universidades del Ministerio de Educación.
} 
importancia de la universidad, mediante un modelo de comunicación que evitara la escasez de información, hecho que genera desconfianza sobre la realidad y posibilidades de las universidades. Una línea de actuación principal debía consistir en realizar acciones centradas en la difusión internacional de las acciones y proyectos de cada universidad, la divulgación científica, o la difusión de su imagen como agente económico (Ministerio de Educación, 2010). Por otro lado, otro de los objetivos de esta estrategia de comunicación consistía en lograr la máxima proyección exterior de su actividad, dotando al denominado Triángulo del conocimiento $^{6}$ de acciones específicas de difusión de la ciencia, mediante la implicación no solo de las universidades, sino también del conjunto de la comunidad universitaria.

En este sentido, podemos decir que la investigación se ha visto beneficiada por la aplicación de las tecnologías para su gestión, optimizando los procesos relacionados con la gestión de la información y la difusión de los resultados. Tal y como se indica en el Informe Universidad 2020 de la Fundación Telefónica (2012: 22): "las TIC se han convertido en una herramienta imprescindible para la difusión de los resultados de la investigación realizada en la Universidad”. En dicho informe, se analiza el impacto de las TIC en los procesos de difusión, midiendo el porcentaje de grupos de investigación que disponen de página web institucional, así como la existencia de aplicaciones web para la inserción de contenidos y gestión de congresos científicos. Los resultados confirman que, a pesar de que se han realizado notables avances en los últimos años, ambas herramientas todavía no han alcanzado una amplia implantación en el ámbito universitario (Fundación Telefónica, 2012).

En cualquier caso, asumiendo que la comunicación es un proceso esencial para el avance de la ciencia y la investigación, y teniendo actualmente a nuestra disposición herramientas de comunicación en línea, resulta fundamental elaborar estrategias basadas en los principios básicos del marketing y la promoción, particularmente en su contexto digital, que permitan aprovechar las posibilidades de esas nuevas herramientas con relación a la difusión, visibilidad y acceso a la actividad científica. En esta misma línea, se manifiestan Seguí Simarro, Poza Luján y Mulet Salort (2015), indicando que resulta esencial realizar un correcto marketing científico. Entre los motivos que avalan dicha afirmación, los autores aluden a aspectos éticos, para comunicar a la sociedad en qué se ha invertido el presupuesto $^{7} \mathrm{y}$, de este modo, poner en relevancia de forma transparente que el gasto en investigación es una inversión. Asimismo, hay que poner en valor el trabajo de los propios investigadores, con el fin de mejorar su imagen pública, su influencia y lograr más visibilidad y reconocimiento de sus resultados de investigación.

Además, la difusión de los trabajos individuales repercutirá directamente sobre la imagen de la universidad como institución. Tal y como manifiesta Sanz

6 Según este informe los factores que constituyen el conocido “Triángulo del Conocimiento” son: la educación, la investigación y la innovación (Ministerio de Educación, 2010: 16).

7 En España, la principal fuente de financiación de la actividad investigadora tiene carácter público (CRUE, 2017). 
Menéndez (2005: 190), “la reputación y el crédito se consiguen por medio de la difusión de los descubrimientos”. Por tanto, no conviene ignorar que las acciones de difusión, marketing y promoción científica ayudan a las organizaciones a potenciar su imagen y competir por recursos económicos.

\section{Objetivos y metodología}

En línea con el contexto de referencia planteado en la introducción, esta investigación tiene como finalidad realizar un análisis comparativo sobre el estado actual en materia de difusión y promoción digital de la investigación a través de las páginas web institucionales de las 50 universidades públicas que forman parte del sistema universitario español y que aparecen en el Registro de Universidades, Centros y Títulos (RUCT) del Ministerio de Educación, Cultura y Deporte ${ }^{8}$.

Se plantean como objetivos específicos conocer qué estrategias se identifican con las líneas de actuación contempladas en los planes y/o documentos estratégicos de estas instituciones y qué recursos se están utilizando para el acceso a dicha información.

En cuanto a la metodología, se trata de un estudio observacional, donde se miden, analizan y evalúan una serie de indicadores previamente definidos, utilizando como método de análisis el proceso de exploración de las páginas web universitarias objeto de estudio y la recogida sistemática y normalizada de datos para, posteriormente, realizar un tratamiento de los mismos para su interpretación por medio de una hoja de cálculo y la ejecución de tablas dinámicas. El estudio se ha desarrollado en el período comprendido entre septiembre de 2017 y enero de 2018.

Los indicadores definidos que han sido objeto de análisis, evaluación y comparación son los siguientes:

- Indicador 1. Menú de investigación: se examina la presencia o no de un menú o apartado específico sobre investigación en la página web principal de cada universidad (variables: valor 1, indica la existencia del menú y valor 0 , no existe).

- Indicador 2. Catálogo y/o base de datos de grupos de investigación: se reconoce la publicación o no de puntos de acceso específicos, catálogos y/o bases de datos de grupos de investigación (variables: valor 1, indica la existencia de un punto de acceso, catálogo y/o bases de datos de grupos de investigación y valor 0 , no existe) y se realiza un estudio de los formatos de presentación existentes para facilitar el acceso a la información sobre su actividad científica.

- Indicador 3. Sistemas de Información sobre Actividad Investigadora (SIAI): verifica la existencia o no de alguna plataforma o portal de investigación, entendidos en este contexto como un SIAI de las universidades (variables: valor 1, existe un SIAI y valor 0, no existe).

8 El RUCT puede consultarse en la dirección: https://www.educacion.gob.es/ruct/home 
- Indicador 4. Proyectos: se analiza la existencia de información o no sobre sobre proyectos de investigación (variables: valor 1, existe información sobre proyectos y valor 0 , no existe). Adicionalmente, se ha explorado la existencia de información o no sobre la financiación de los proyectos de investigación y su procedencia. En este sentido, dado que gran parte de la financiación es pública, analizar este elemento implica un reconocimiento a la transparencia que debe ser exigida a dichos proyectos. Del mismo modo, la existencia de dicha información puede servir como base para la observación de la inversión realizada por cada institución y qué universidades proporcionan herramientas adicionales para el acceso y recuperación de este tipo de información.

- Indicador 5. Recursos y portales bibliométricos: se ha comprobado la existencia de iniciativas/recursos ad hoc de elaboración sintética y visual de datos relacionados con la productividad científica y la actividad investigadora desarrollados para potenciar la reputación de la institución a partir de la explotación de sus resultados de investigación (variables: valor 1, existen recursos y portales bibliométricos y valor 0 , no existe).

Como colofón del estudio, se ha realizado un análisis de la existencia de iniciativas pioneras en la difusión y promoción digital de la investigación orientadas a reforzar sus estrategias en este contexto, así como una breve reflexión sobre la visibilidad de los repositorios digitales y recursos gestionados por las bibliotecas universitarias en relación con los objetivos del trabajo.

También se han analizado las líneas de actuación, específicamente referidas a investigación, recogidas en los planes y documentos estratégicos formulados por las universidades objeto de análisis, de lo que se puede inferir el grado de importancia que las universidades conceden a la difusión digital de la investigación. Específicamente y, con el fin de perfilar tendencias de futuro en esta línea, se ha realizado un análisis de contenido de los planes estratégicos disponibles en las páginas web de las universidades que presentan un alcance temporal posterior al año 2017. No obstante, esta línea de asociación entre los planes estratégicos y su relación con otras posibles variables queda abierta para su estudio en líneas futuras de trabajo.

\section{Resultados y discusión}

\subsection{Menú “Investigación”}

El primero de los parámetros que nos interesaba analizar era la presencia de un menú en la página web principal de cada universidad bajo la denominación de "Investigación” o términos similares, que aglutine la consulta de todos los aspectos relacionados con la investigación en cada una de las universidades objeto de análisis. Si bien el titular más común de dicho menú es el término “Investigación”, existen alternativas como "Investigación y transferencia” o " $\mathrm{I}+\mathrm{D}+\mathrm{i}$ ", entre otras. Específicamente, de las 50 universidades analizadas, 47 dispone de un menú 
específico con dicha denominación; 2 de ellas carecen de un apartado concreto que englobe las cuestiones sobre investigación, y, por último, una de ellas incorpora la información referente a investigación en un menú denominado “empresa”.

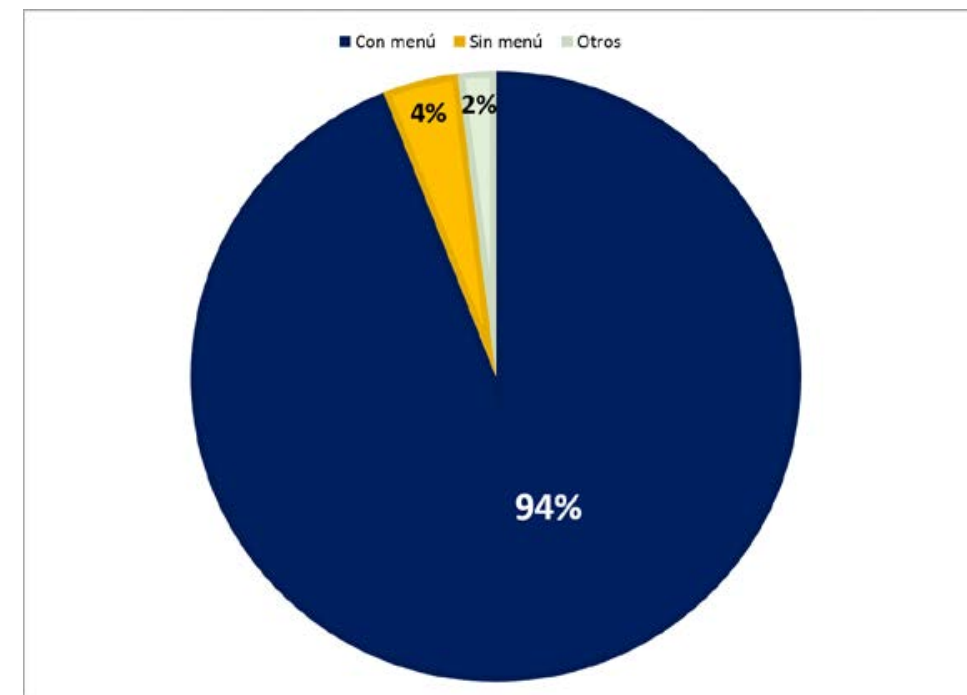

Figura 1. Indicador 1. Distribución de universidades según la existencia/ausencia de un menú de investigación (en porcentajes).

No obstante, a pesar de que de un análisis preliminar de los datos anteriores se puedan derivar esfuerzos en la línea de visibilizar y difundir la actividad investigadora en el contexto digital, durante el propio desarrollo de la investigación se ha observado cierta falta de sistematización de la información recogida en este menú de investigación, encontrando mucha información de forma dispersa y, en cierto modo, poco organizada. La ausencia de una clasificación de la información orientada a los posibles usuarios y partes interesadas (propia comunidad académica, público externo, empresas, etc.) dificulta el acceso a los recursos destinados a la difusión de la investigación y, por lo tanto, a la información.

\subsection{Catálogos y/o bases de datos de grupos de investigación}

En el contexto universitario, los grupos de investigación son definidos como la unidad básica y fundamental para la generación de conocimiento científico y la Ley Orgánica de Universidades (LOU) los contempla como la estructura principal, además de los institutos de investigación, desde la que se lleva a cabo la investigación (artículo 40).

Si bien las universidades cuentan con institutos y centros de investigación, estos suelen ser menos numerosos, y para conocer su actividad desde la página web puede resultar suficiente con un listado o directorio. No ocurre lo mismo con los grupos de investigación que, tal y como se ha señalado en el apartado de introducción (CRUE, 2017: 106), son numerosos en todas las universidades 
analizadas. Este hecho hace muy recomendable la creación de plataformas de diverso carácter que faciliten el acceso a la información sobre su actividad científica.

En esta línea, se ha de poner de manifiesto que el 88\% de las universidades cuentan con algún tipo de punto de acceso, catálogo y/o base de datos que incluye la información disponible sobre sus grupos de investigación; encontramos un $12 \%$ de instituciones en las que no existe un punto común de acceso a la información sobre los grupos, o en el caso de existir, no funciona (figura 2).

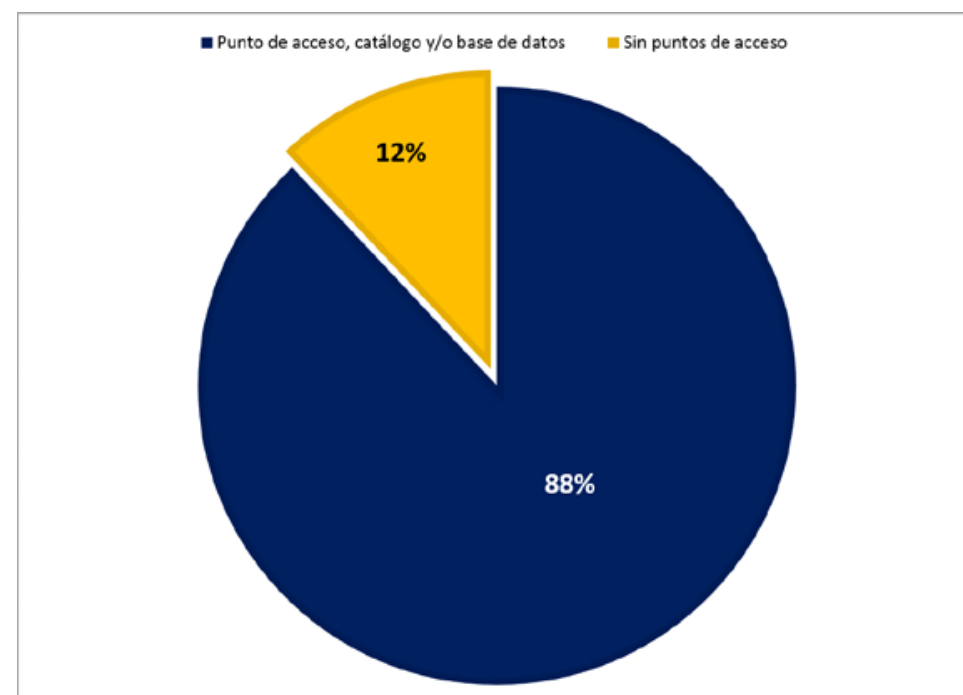

Figura 2. Indicador 2. Distribución de universidades según la existencia/ausencia de puntos de acceso a la información sobre grupos de investigación (en porcentajes)

Un análisis detallado de la información presentada en la figura 2, en lo referido específicamente a las universidades que disponen de algún punto de acceso a la información disponible sobre grupos de investigación en las universidades objeto de estudio, nos permite establecer la siguiente sistematización de los formatos de presentación de la información en esos casos: 
Tabla 1. Clasificación de los formatos de presentación en cuanto a puntos de acceso a la información

\begin{tabular}{|l|l|}
\hline Grupo 1 & Documentos con relación de nombres \\
\hline Grupo 2 & $\begin{array}{l}\text { Índices o relaciones de grupos de investigación clasificados por } \\
\text { criterios }\end{array}$ \\
\hline Grupo 3 & $\begin{array}{l}\text { Índices clasificados por criterios con combinación de interfaces } \\
\text { de búsqueda simple }\end{array}$ \\
\hline Grupo 4 & $\begin{array}{l}\text { Índices clasificados por criterios con combinación de interfaces } \\
\text { de búsqueda avanzada }\end{array}$ \\
\hline
\end{tabular}

Grupo 1: Documentos con relación de nombres

Grupo 2: Índices o relaciones de grupos de investigación clasificados por criterios.

Este grupo de universidades facilita el acceso a la información sobre sus grupos de investigación mediante la relación de sus nombres clasificados según uno o varios criterios: alfabéticamente, por departamentos, por facultades o por áreas o campos generales de conocimiento. En este grupo se incluyen la mayoría de las universidades, pues la elaboración de índices o relaciones clasificados por campos generales de conocimiento es la opción más recurrente, con el 34\% de los casos del total. Ejemplo de este grupo sería la Universidad de Granada, como se muestra en la figura 3.

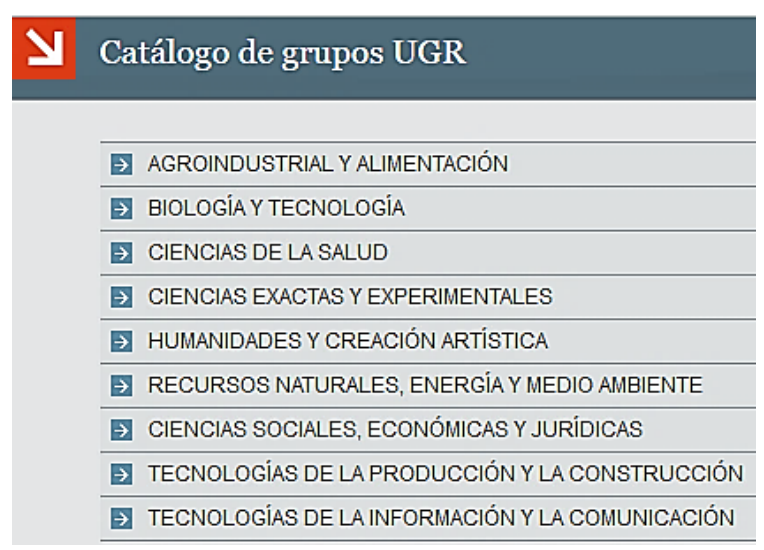

Figura 3. Catálogo de grupos de investigación de la Universidad de Granada

Grupo 3: Índices clasificados por criterios con combinación de interfaces de búsqueda simple.

En este grupo englobamos las universidades que, además de ofrecer los índices o relaciones clasificados bajo algún criterio de los mencionados en el apartado anterior, también permiten la búsqueda simple mediante algún filtro: palabra clave, nombre, línea de investigación, facultad, departamento o sector de actividad. 
Forman parte de este grupo las siguientes universidades: Autónoma de Barcelona, Jaén, Valencia, Politécnica de Cataluña, Coruña, León, Girona, Complutense de Madrid, Cantabria e Islas Baleares (figura 4).

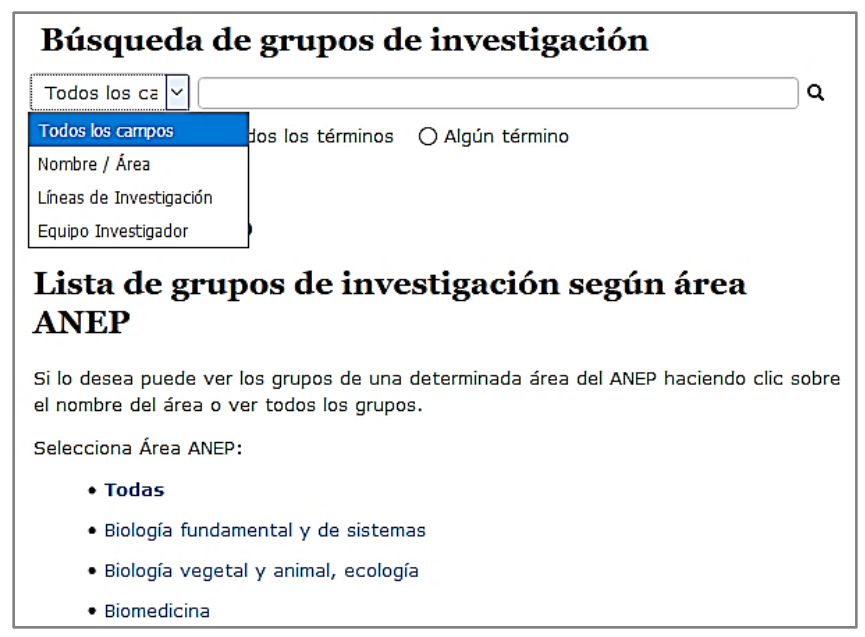

Figura 4. Búsqueda de grupos de investigación de la Universidad de las Islas Baleares

Grupo 4: Índices clasificados por criterios con combinación de interfaces de búsqueda avanzada.

Por último, identificamos una serie de universidades que facilitan el acceso a la información de sus grupos mediante la relación de los nombres clasificados según uno o varios criterios y, además, existe una opción de búsqueda avanzada. Estas opciones de búsqueda pueden ser consideradas como buenas prácticas pues facilitan el acceso y recuperación de la información de los grupos de investigación por las partes interesadas.

Este grupo está compuesto por la Universidad de Castilla la Mancha, Universidad de las Palmas de Gran Canaria, Universidad Politécnica de Madrid, Universidad de Zaragoza, Universidad de Barcelona, Universidad de Lleida, Universidad de Santiago de Compostela y Universidad de Murcia (figura 5). 


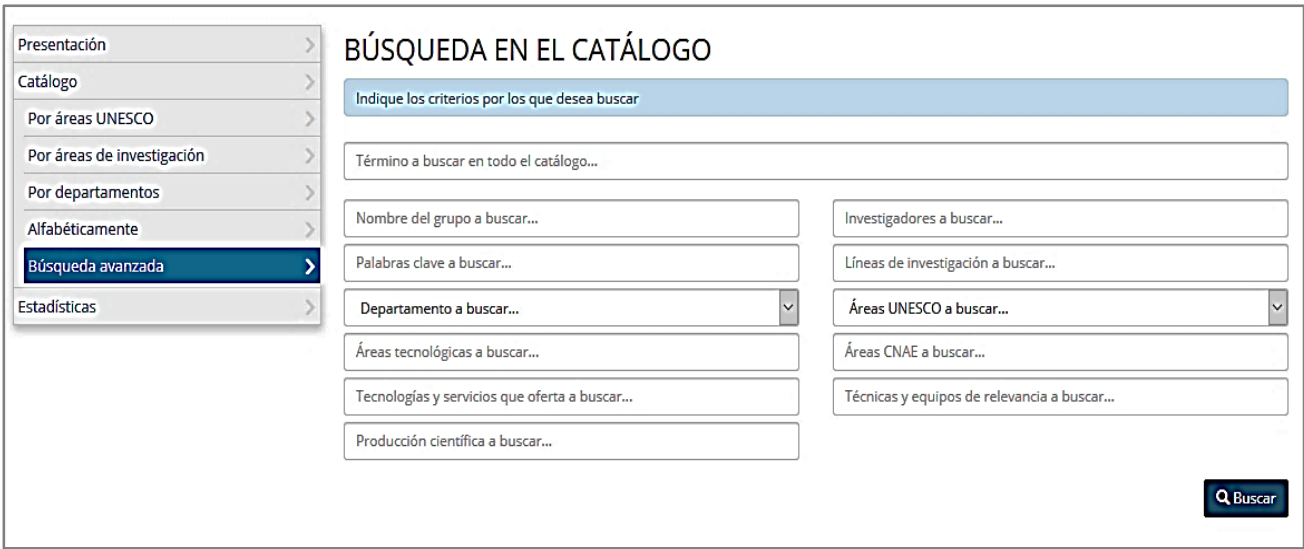

Figura 5. Catálogo de grupos de investigación de la Universidad de Murcia

A modo de resumen de lo expuesto en relación con este indicador, sintetizamos a continuación la distribución porcentual de los diferentes formatos de presentación de la información disponible sobre grupos de investigación en las universidades objeto de análisis (figura 6).

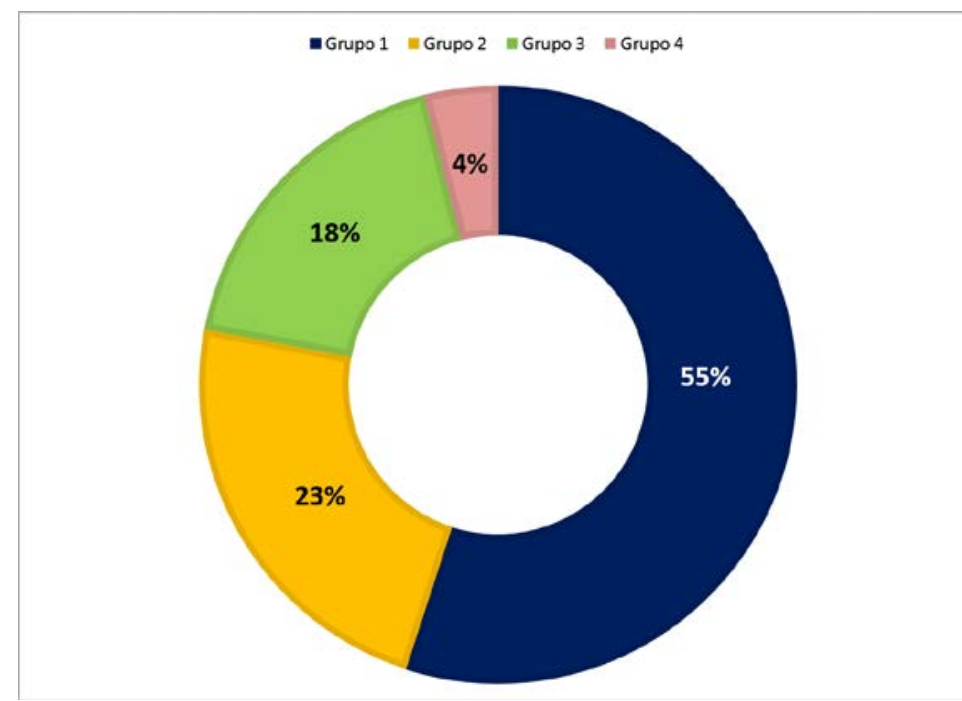

Figura 6. Formatos de presentación de la información disponible sobre grupos de investigación (en porcentaje) 


\subsection{Sistemas de Información sobre Actividad Investigadora (SIAI)}

Tal y como se ha definido en la metodología, otro de los indicadores esenciales para medir el grado de difusión digital de la actividad científica se relaciona con la verificación de la existencia de algún tipo de plataforma o portal de investigación, entendidos en este contexto como SIAI ${ }^{9}$ que también permiten en muchos casos el acceso a los resultados de investigación generados por las universidades. En línea con dicho concepto, se han analizado las diversas iniciativas que han o están desarrollando las universidades para dar visibilidad a sus resultados de investigación.

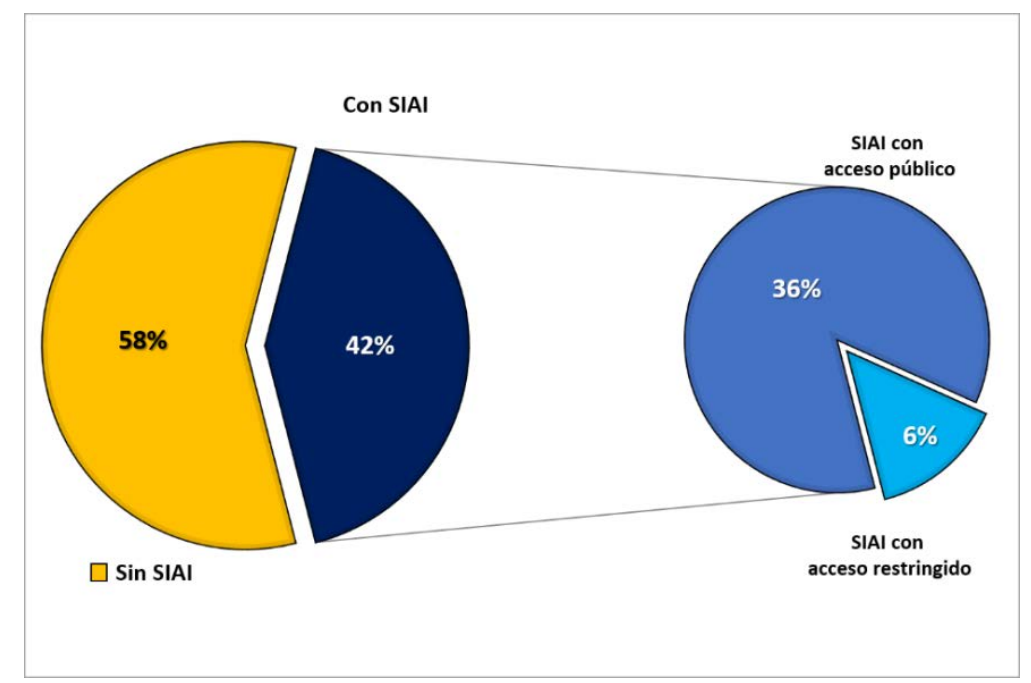

Figura 7. Indicador 3. Presencia de SIAI por universidades y tipos de acceso (en porcentajes)

De las 50 universidades objeto de estudio, el 42\% cuenta con algún tipo de base de datos, de las cuales el $36 \%$ es de acceso público permitiendo la búsqueda de resultados de la investigación o la actividad científica desarrollada en la institución, mientras que el 6\% restante es de acceso restringido (figura 7).

Es importante destacar que en este punto tampoco encontramos una normalización en el tipo de portales sobre investigación que las universidades implementan para el fin descrito, sino que existen diversas opciones, siendo la más común la plataforma de gestión de la investigación desarrollada por la Universidad de Barcelona (GREC), y que actualmente utilizan también otras instituciones.

A continuación, mostramos en la tabla 2 la distribución de sistemas de información sobre investigación y/o producción científica por universidades:

\footnotetext{
9 Conceptualmente relacionados con el término en inglés Current Research Information System (CRIS).
} 
Tabla 2. Sistemas de información por universidades

\begin{tabular}{|c|c|c|}
\hline SIAI & UNIVERSIDAD & URL \\
\hline \multirow{4}{*}{$\begin{array}{l}\text { Portal de producción } \\
\text { científica }\end{array}$} & $\begin{array}{lll}\text { Universidad } & \text { Autónoma } & \text { de } \\
\text { Barcelona } & & \\
\end{array}$ & https://goo.gl/agz66y \\
\hline & $\begin{array}{l}\text { Universidad Autónoma de } \\
\text { Madrid }\end{array}$ & $\begin{array}{l}\underline{\mathrm{https}: / / \text { portalcientifico.uam.e }} \\
\underline{\mathrm{s} /}\end{array}$ \\
\hline & Universidad Pompeu Fabra & $\begin{array}{l}\text { https://producciocientifica.u } \\
\text { pf.edu }\end{array}$ \\
\hline & Universidad de Navarra & https://goo.gl/Qpg8Fx \\
\hline UC3M Research Portal & $\begin{array}{llll}\text { Universidad } & \text { Carlos } & \text { III } & \text { de } \\
\text { Madrid } & & & \\
\end{array}$ & $\begin{array}{l}\underline{\text { https://researchportal.uc3m.e }} \\
\underline{\mathrm{s} /}\end{array}$ \\
\hline Portal Bibliométrico $^{10}$ & $\begin{array}{l}\text { Universidad Complutense de } \\
\text { Madrid }\end{array}$ & $\begin{array}{l}\text { https://bibliometria.ucm.es/i } \\
\text { nicio }\end{array}$ \\
\hline Búsqueda avanzada & Universidad de Almería & https://goo.gl/dRKUVW \\
\hline $\begin{array}{l}\text { Buscador } \\
\text { investigación }\end{array}$ & Universidad de Extremadura & https://goo.gl/HG9jDo \\
\hline \multirow{5}{*}{ GREC } & Universidad de Barcelona & https://webgrec.ub.edu/ \\
\hline & Universidad de Girona & https://webgrec.udg.edu/ \\
\hline & Universidad de Lleida & www.udl.cat/ca/recerca/grec \\
\hline & Universidad Rovira i Virgili & https://webgrec.urv.es \\
\hline & $\begin{array}{l}\text { Universidad de las } \\
\text { Baleares }\end{array}$ & https://webgrec.uib.es/ \\
\hline UGR Investiga & Universidad de Granada & $\begin{array}{l}\text { http://investigacion.ugr.es/ug } \\
\text { ninvestiga/ }\end{array}$ \\
\hline UR Investiga & Universidad de la Rioja & $\begin{array}{l}\text { http://investigadoresur.unirio } \\
\text { ja.es/investigadoresur/ }\end{array}$ \\
\hline SISIUS & Universidad de Sevilla & $\begin{array}{l}\text { https://investigacion.us.es/sis } \\
\text { ius }\end{array}$ \\
\hline Futur & $\begin{array}{lll}\text { Universidad } & \text { Politécnica } & \text { de } \\
\text { Cataluña } & & \end{array}$ & https://futur.upc.edu/ \\
\hline Observatorio I+D+i & $\begin{array}{l}\text { Universidad Politécnica de } \\
\text { Madrid }\end{array}$ & $\begin{array}{l}\text { http://www.upm.es/observat } \\
\text { orio/vi/index.jsp }\end{array}$ \\
\hline
\end{tabular}

Aunque la presencia de este tipo de plataformas en las webs de las universidades no está generalizada aún, sí se pone de manifiesto la importancia de mostrar públicamente la información sobre producción en investigación, tal y como se desprende del surgimiento de iniciativas más recientemente impulsadas por algunas de estas instituciones, como sería el caso de los portales de la Universidad Carlos III y la Universidad Complutense.

Además, estas iniciativas son apoyadas por determinadas comunidades autónomas que, con la finalidad de dar visibilidad al potencial de sus instituciones, crean bases de datos y portales científicos. Sería el caso de Andalucía y su Sistema

10 Además de proporcionar información bibliométrica, en este caso configura también el punto de acceso a la actividad investigadora producida en la Universidad Complutense de Madrid 
de Información Científica de Andalucía (SICA) o Cataluña y el Portal de la Recerca de les Universitat de Catalunya (PRC).

\subsection{Difusión de la investigación sobre proyectos de investigación y su financiación}

En el cumplimiento de su función de investigación, las universidades llevan a cabo la actividad investigadora mediante proyectos, resultado de convocatorias de financiación de las propias universidades, de las comunidades autónomas y/o de las convocatorias de financiación de carácter nacional e internacional. En el marco de estos proyectos surge una gran cantidad de producción científica y resultados de investigación.

Dado que la explotación de los sistemas de información vistos en el apartado anterior puede resultar compleja y en ocasiones poco precisa, motivada por los conocidos problemas de normalización, es fundamental la elaboración y disposición de herramientas adicionales y/o complementarias, que pueden integrarse en los sistemas de información a los que nos hemos referido en el apartado anterior, que faciliten el acceso y recuperación de la información específica sobre proyectos de investigación.

En esta línea, tan solo el $22 \%$ de las universidades facilitan específicamente información sobre sus proyectos de investigación a través de un punto de acceso común.

Nuevamente observamos que la disposición de este tipo de contenido es diferente según las universidades y cómo las diversas opciones facilitan o dificultan la recuperación y acceso a esta información específica.

Detallamos, a continuación, las posibilidades de búsqueda y un cuadro resumen (figura 8):

- Universidad de Castilla la Mancha (UCLM): Listados clasificados por proyectos regionales, nacionales e internacionales. Se trata de proyectos finalizados a modo de memoria de investigación.

- Universidad Carlos III (UC3M): Listados de proyectos internacionales activos clasificados por programas, con enlaces a la página web de los mismos.

- Universidad de Huelva (UHU): Listados clasificados por proyectos nacionales e internacionales y, a su vez, por programas. No se especifica si son proyectos activos o finalizados.

- Universidad de León (UNILEON): Listado de proyectos internacionales clasificados por programas, con enlaces a las webs. No se especifica si son proyectos activos o finalizados.

- Universidad de Oviedo (UNIOVI): Listado de proyectos internacionales clasificados por campos de conocimiento. Entre paréntesis se especifica el alcance temporal y enlaza a las webs. 
- UNED: Listados de proyectos de investigación nacionales clasificados por campos de conocimiento y facultades. En cada facultad la ordenación es cronológica y la información es básica, no enlazando con las webs.

- Universidad Politécnica de Cartagena (UPCT): Listado de varias páginas sin posibilidad de búsqueda. En algunos casos enlaza con la web del proyecto.

- Universidad Pompeu Fabra (UPF): Proyectos europeos clasificados por programas y subprogramas. Se especifica el alcance temporal y enlaza a webs.

- Universidad Rey Juan Carlos (URJC): Dentro de la sección de proyectos vivos permite seleccionar los proyectos autonómicos, estatales e internacionales y, a su vez, los clasifica por campos de conocimiento. Enlaza a webs.

- Universidad de las Islas Baleares (UIB): Listados por proyectos autonómicos, estatales e internacionales y, a su vez, diferencia los proyectos vigentes de los finalizados recientemente. Enlace a webs.

- Universidad de Barcelona (UB): Posibilidad de búsqueda por proyectos nacionales o europeos y por palabra clave. Además, ofrece un listado alfabético. Aunque no lo especifique, se trata de proyectos activos, pero no enlaza a las webs de los mismos.

\begin{tabular}{|l|r|r|r|r|r|r|r|r|r|}
\cline { 2 - 9 } & \multicolumn{3}{c|}{ TIPO DE PROYECTOS } & \multicolumn{2}{c|}{ BÚSQUEDA } & \multicolumn{2}{c|}{ VIGENCIA } & \multicolumn{2}{c|}{ INFORMACIÓN } \\
\cline { 2 - 9 } & Autonómico & \multicolumn{1}{c|}{ Nacional } & Internacional & Sencilla & Listados & Activos & Finalizados & Básica & Webs \\
\hline UCLM & 1 & 1 & 1 & 0 & 1 & 0 & 1 & 1 & 0 \\
\hline UC3M & 0 & 0 & 1 & 0 & 1 & 1 & 0 & 1 & 1 \\
\hline UHU & 0 & 1 & 1 & 0 & 1 & 0 & 0 & 1 & 0 \\
\hline UNILEON & 0 & 0 & 1 & 0 & 1 & 0 & 0 & 1 & 1 \\
\hline UNIOVI & 0 & 0 & 1 & 0 & 1 & 0 & 0 & 1 & 1 \\
\hline UNED & 0 & 1 & 0 & 0 & 1 & 0 & 0 & 1 & 0 \\
\hline UPCT & 0 & 0 & 0 & 0 & 1 & 0 & 0 & 1 & 1 \\
\hline UPF & 0 & 0 & 1 & 0 & 1 & 0 & 0 & 0 & 1 \\
\hline URJC & 1 & 1 & 1 & 0 & 1 & 1 & 0 & 1 & 1 \\
\hline UIB & 1 & 1 & 1 & 0 & 1 & 1 & 1 & 0 & 1 \\
\hline UB & 0 & 1 & 1 & 1 & 1 & 1 & 0 & 1 & 0 \\
\hline Total & 3 & 6 & 9 & 1 & 11 & 4 & 2 & 9 & 7 \\
\hline
\end{tabular}

Figura 8. Indicador 4. Universidades por tipo de información sobre de proyectos.

\subsection{La investigación en cifras: recursos y portales bibliométricos}

Con el fin de potenciar la reputación de la institución mediante la investigación, las universidades, fundamentalmente a través de sus bibliotecas universitarias y unidades de bibliometría, están desarrollando iniciativas ad hoc de elaboración sintética y visual de los datos relacionados con su productividad científica y su actividad investigadora. En este punto, destacamos específicamente aquellas iniciativas directamente relacionadas con el fin descrito: 
- Universidad Carlos III de Madrid: Portal muy completo con indicadores que recogen toda su actividad investigadora durante un periodo de dos años. El portal $^{11}$ se compone de distintos contenidos en formato multimedia (vídeo, bases de datos, gráficos...) estando disponible en la web para todo el público.

- Universidad de Cádiz: Desde el portal de la Unidad de Bibliometría ${ }^{12}$ se puede navegar por diversos indicadores de investigación, informes bibliométricos y memorias de investigación.

- Universidad de Granada: El proyecto Livemetrics ${ }^{13}$ muestra de forma atractiva, dinámica y actualizada los indicadores bibliométricos y las estadísticas de $\mathrm{I}+\mathrm{D}$ de la Universidad mediante tres grupos de indicadores calculados a través de la Web of Science: producción de trabajos internacionales, impacto en términos de citación y colaboración científica.

- Universidad Complutense de Madrid: El Portal Bibliométrico ${ }^{14}$ tiene como objetivo visibilizar la producción científica de la universidad mediante la difusión y acceso público a la información bibliométrica de todos los/as profesores/as disponible en las bases de datos Scopus y/o Google Scholar.

- Universidad Politécnica de Cataluña: En su portal de producción científica FUTUR $^{15}$ mantiene una cabecera actualizada con los datos más relevantes sobre los investigadores, publicaciones, proyectos, etc.

\subsection{Iniciativas pioneras y repositorios institucionales}

a) Iniciativas pioneras.

En línea con lo comentado anteriormente en relación con la creación de herramientas adicionales que faciliten el acceso a la información sobre investigación, varias universidades disponen en línea de guías o áreas de expertos que permiten acceder fácilmente al trabajo de sus académicos.

Un ejemplo sería la Universidad de Huelva, que mediante su catálogo de expertos ofrece varias posibilidades para localizar a los investigadores: áreas ANEP, líneas de investigación o búsqueda por palabra clave.

Referente a las redes sociales, la Universidad de Granada ha creado recientemente una nueva aplicación que permite acceder y consultar los datos de los investigadores que se encuentren vinculados a sus grupos de investigación y que tengan perfiles creados en las siguientes redes sociales académicas: Researchgate, Mendeley, Publons, Academia.edu y Dialnet.

Por otro lado, la Universidad Politécnica de Cataluña cuenta con el proyecto GEOCommons, con el que pretende mostrar el impacto que la universidad tiene a lo largo de todo el mundo con sus publicaciones académicas, basado en los documentos académicos depositados en el repositorio institucional UPCommons, portal del conocimiento abierto de la UPC.

\footnotetext{
https://hosting01.uc3m.es/InvestigaUc3m/2015-2016/carlos3/es/ [Consulta: 27/11/2017]

http://bibliometria.uca.es/ [Consulta: 27/11/2017]

livemetrics.ugr.es/ [Consulta: 27/11/2017]

https://bibliometria.ucm.es/inicio [Consulta: 27/11/2017]

https://futur.upc.edu/ [Consulta: 27/11/2017]
} 
Por último, el UPF Knowlegde Portal, un portal en inglés que funciona como ventana única para acceder a los resultados y capacidades de investigación de la Universidad Pompeu Fabra relacionados con la innovación y el emprendimiento, que permite la búsqueda de patentes, tecnologías, grupos de investigación o spin offs ${ }^{16}$, con el objetivo principal de colaborar con sus proyectos de I+D+i.

b) Repositorios digitales institucionales.

Las bibliotecas universitarias son una pieza clave para el impulso y desarrollo de este tipo de iniciativas. Aunque los portales bibliométricos son más recientes, no cabe duda de que los repositorios institucionales constituyen desde hace tiempo el instrumento por excelencia para la preservación, difusión y visibilidad de la producción científica, proporcionando acceso abierto no solo a la información científica, sino también a los propios contenidos.

Esta afirmación es acertada teniendo en cuenta que todas las universidades analizadas tienen un repositorio institucional digital. Por el contrario, y en la línea de lo comentado en el punto anterior, tan solo el $28 \%$ de las universidades hacen accesible fácilmente el repositorio desde las páginas principales del sitio web.

Una situación similar ocurre con las bibliotecas, las unidades de 112ibliometría y los recursos que producen. Todos estos factores limitan, por un lado, la visibilidad de las bibliotecas y su implicación en la generación de recursos imprescindibles para el acceso a la información investigadora y, por otro, el propio acceso a los resultados de investigación.

\subsection{Planes estratégicos y tendencias}

De todas las universidades analizadas, el 58\% permite la consulta a través de la web de documentos o planes estratégicos que incluyen acciones futuras en relación con la investigación y que presentan un alcance temporal posterior a $2017^{17}$. El $42 \%$ restante se corresponde con universidades que no tienen disponible el plan estratégico o que su alcance temporal finalizó con anterioridad al 2018 y que, por tanto, no han sido analizadas, dado que el objeto de este epígrafe es perfilar las tendencias de futuro en este contexto.

Del 58\% mencionado, la mayoría de universidades consideran entre sus ejes estratégicos acciones de comunicación, difusión y visibilidad de la actividad investigadora, lo que se traduce en la relevancia de estas acciones y la creciente importancia que las instituciones conceden a este tipo de iniciativas.

Podemos clasificar en un primer grupo aquellas universidades que, de forma generalizada, contemplan actuaciones relacionadas con la potenciación de la difusión y la visibilidad de la investigación. Estas serían la Universidad Autónoma

16 Iniciativas empresariales cuya actividad se basa en la explotación de nuevos procesos, productos o servicios a partir del conocimiento adquirido y los resultados obtenidos en la propia Universidad. Fuente: Spin-offs Universidad de Granada [http://spinoff.ugr.es]. Para una información más detallada sobre el concepto y tipologías de spin-offs universitarias se puede consultar el trabajo de: Pirnay, Surlemont y Nlemvo (2003).

17 En el Anexo 1 se presenta una relación de los planes estratégicos con un alcance temporal posterior a 2017 que han sido consultados y que están disponibles a través de las páginas web de las universidades. 
de Madrid, la Universidad de Alcalá, la Universidad de Alicante, la Politécnica de Valencia, la Universidad de Extremadura, la Universidad de Almería y la Universidad de Santiago de Compostela, que prevén estrategias en esta línea. Por su parte, la Universidad de Sevilla, a través de la Unidad de Bibliometría, pretende incrementar el impacto, la visibilidad y la difusión de los resultados de su investigación.

Más relacionado con cuestiones de visibilidad, la Universidad Pompeu Fabra quiere velar por la visibilidad de los resultados de la investigación mediante la búsqueda de las mejores fórmulas, por ejemplo, por medio de la difusión de experiencias de éxito de los grupos de investigación, estrategias proactivas de comunicación y otras medidas dinamizadoras.

Por su parte, las Universidades de Coruña, León y Valencia consideran el desarrollo de acciones para potenciar e incrementar la visibilidad de la actividad investigadora.

Por último, identificamos otro grupo de universidades que detallan en sus planes estratégicos acciones concretas sobre difusión en el contexto digital.

La Universidad Carlos III de Madrid, con el objetivo final de compartir y descubrir todos los datos en Internet para visibilizar la ciencia realizada en la universidad, ya ha ido realizando alguna de las acciones previstas en el plan, como la creación de una página de información de la investigación y la publicación de un portal de investigación que proporciona acceso a la información científica y de investigación.

La Universidad Complutense también ha concretado alguna de sus acciones, como la creación de la Unidad de Bibliometría y el Portal Bibliométrico, y entre las acciones futuras prevé la creación de una aplicación similar a la que tiene la Universidad de Granada.

La implantación de canales de comunicación para promover la difusión pública de los resultados de la actividad investigadora también está prevista por la Universidad de Jaén y la Universidad de la Laguna. Esta última, además, fomentará la autodifusión mediante webs, blogs, repositorios, etc.

Visibilizar la producción de los investigadores mediante la difusión de la investigación a través de la web institucional y de las unidades de investigación con el apoyo de la Unidad de Bibliometría es también un objetivo principal para la Universidad de las Palmas de Gran Canaria.

Por su parte, la Universidad de Oviedo prevé la creación de un sistema de difusión de la actividad investigadora y de la transferencia desarrollada, además de la elaboración de un plan de difusión renovado anualmente.

En la misma línea, la Universidad de Salamanca considera potenciar la imagen institucional mediante la creación de un sistema de comunicación institucional que favorezca la difusión de las actividades, líneas, resultados y avances de los grupos de investigación, así como de su oferta tecnológica.

La Universidad Rovira i Virgili tiene entre sus objetivos promover el reconocimiento de la investigación y la innovación mediante, entre otros criterios, la difusión y visibilidad de la actividad I+D+i en la web y las redes sociales. 
La Universidad de Valladolid, en las Directrices Generales de Política Docente e Investigadora 2017-2018, informa sobre la puesta en marcha de un sistema de información que permita la difusión de los resultados de la investigación y ayude a crear una cultura científica que trascienda los límites de la universidad.

\section{Conclusiones}

Son diversas las causas que llevan a las universidades a realizar acciones de difusión digital con la intención de mostrar públicamente y facilitar el acceso a la información relacionada con la investigación: optimizar el trabajo realizado, reforzar los procesos de gestión de los datos de investigación, dar visibilidad a la potencialidad de la institución, incrementar la visibilidad y acceso al trabajo de los investigadores, mejorar la imagen pública y reputación, consolidarse como marca institucional y, en última instancia, aumentar las opciones de obtención de financiación.

Para ello, llevan a cabo diversas estrategias y desarrollan herramientas online que facilitan el acceso a la información sobre investigación.

La metodología utilizada permite un acercamiento preliminar y propio de una fase de análisis del objeto de estudio, haciéndose imprescindible una revisión de forma progresiva en futuras líneas de investigación. Esta revisión debe facilitar otros posibles resultados a partir del estudio de un mayor número de variables y la relación entre las mismas. Del mismo modo, hay que considerar que algunos de los indicadores de tipo binario propuestos pueden exigir ciertas consideraciones de mejora, para poder aportar una visión de los resultados con un mayor alcance, apoyando su comprensión e interpretación con mayor grado de detalle. En cualquier caso, hay que valorar que estos indicadores iniciales nos ofrecen una primera aproximación que puede constituir una base metodológica para su sistematización y su ampliación podría ser objeto de desarrollos posteriores

No obstante, el estudio realizado nos permite establecer que no existe una situación homogénea en las universidades públicas del Sistema Universitario español y que, a pesar de algunos puntos débiles que se deberían mejorar, existen bastantes iniciativas que permiten pronosticar el creciente interés de las instituciones por este tipo de estrategias.

Referente a las debilidades, se podrían introducir mejoras en la estructuración del menú investigación, de modo que permita identificar los recursos, productos y servicios según el tipo de usuario, así como una mayor conexión con los repositorios institucionales y recursos generados por las bibliotecas universitarias como unidades fundamentales en el apoyo a la difusión de la investigación.

De los recursos analizados, son los catálogos o relaciones de grupos de investigación las herramientas más comunes de encontrar en las webs universitarias. En este sentido, la difusión tanto a escala nacional como internacional de las capacidades y potencial de los grupos de investigación es un aspecto clave para lograr una mayor proyección y obtener mejor financiación de la institución en su conjunto. 
Por el contrario, los demás recursos analizados, como los portales de investigación y la información sobre proyectos de investigación financiados, no cuentan con tanta aceptación y los podemos localizar en pocas universidades.

Son necesarios esfuerzos en la creación de herramientas realmente eficaces en la recuperación de la información mediante acciones y procesos de normalización, formatos estándares y protocolos de interoperabilidad, el uso de identificadores como ORCID que eviten ambigüedades, así como favorecer el acceso abierto a la producción científica y el uso de los repositorios institucionales.

La situación es positiva en cuanto a iniciativas de creación de portales bibliométricos, impulsados por las bibliotecas universitarias y la creación de unidades de bibliometría, a las que se les concede cada vez más importancia.

La no disponibilidad de planes estratégicos o de planes cuyo alcance temporal tiene fecha próxima de finalización, evidencia que el sistema universitario se encuentra en un momento de cambio y modernización. En varias de las universidades analizadas se informa de la elaboración de nuevos planes estratégicos.

Además, las líneas de actuación de los planes estudiados constatan la existencia de futuras estrategias de difusión digital de la investigación.

En cualquier caso, se pone de manifiesto la importancia de una orientación basada en el marketing, en el sentido de hacer más accesible la información y los propios contenidos, lo que repercute positivamente en la imagen, la marca y la reputación de la institución.

\section{Referencias Bibliográficas}

Cegarra Sánchez, J. (2012). La difusión de la investigación. Madrid: Ediciones Díaz de Santos

Crue (2017). La universidad española en cifras 2015/2016. ISBN 978-84-697-6269-1

España (2001). Ley Orgánica 6/2001, de 21 de diciembre, de Universidades. Boletín Oficial del Estado, 24 de diciembre de 2001, 307, pp. 49400-49425. <https://www.boe.es/boe /dias/2001/12/24/pdfs/A49400-49425.pdf>. [Consulta: 15/11/2016].

España (2011). Ley 14/2011, de 1 de junio, de la Ciencia, la Tecnología y la Innovación. Boletín Oficial del Estado, 2 de junio de 2011, 131, pp. 54387-54455. $<$ https://www.boe.es/boe/dias/2011/06/02/pdfs/BOE-A-2011-9617.pdf>. [Consulta: $16 / 11 / 2016]$.

Espinosa Santos, V. (2010). Difusión y divulgación de la investigación científica. IDESIA, v.28, n.3. http://dx.doi.org/10.4067/S0718-34292010000300001

Fundación Telefónica (2012). Universidad 2020: papel de las TIC en el nuevo entorno socioeconómico. ISBN: 978-84-08-11014-9

Ministerio De Educación (2010). Estrategia universidad 2015: contribución de las universidades al progreso socioeconómico español 2010-2015. Madrid: Ministerio de Educación de España

Pérez-Montoro, M. (2014). Políticas universitarias de difusión de la información a través de la propia web institucional. El profesional de la información, 23(2), 190-194. http://dx.doi.org/10.3145/epi.2014.mar.12 
Pinto Molina, M.; Alonso Berrocal, J.L.; Cordón García, J.A.; Fernández Marcial, V.; García Figuerola, C.; García Marco, J.; Gómez Camarero, C.; Zazo, A.F., Doucet, A.V. (2004). Análisis cualitativo de la visibilidad de la investigación de las universidades españolas a través de sus páginas web. Revista Española de Documentación Científica, 23(3), 345-370. doi:10.3989/redc.2004.v27.i3.157.

Pirnay, F.; Surlemont, B. Y Nlemvo, F. (2003). Toward a typology of university spin-offs. Small Business Economics, 21(4): 355-369.

Rauhvargers, A. (2013). Global University rankings and their impact. Report II. EUA Reports on rankings 2013. Brussels: European University Association.

Sanz Menéndez, L. (2005). Universidad e investigación: la financiación competitiva de los proyectos de $\mathrm{I}+\mathrm{D}$, con especial referencia a las Ciencias Sociales y Económicas. Reis, 109/05, 181-218

Saraite-Sariene, L; Gálvez Rodríguez, Mm y Haro De Rosario, A. (2018). Exploring determining factors of web transparency in the world's top universities. Revista de Contabilidad - Spanish Accounting Review, 21(1), 63-72

Seguí Simarro, Jm; Poza Luján, Jl y Mulet Salort, Jm. (2015). Estrategias de divulgación científica. Valencia: Editorial de la Universidad Politécnica de Valencia

\section{ANEXO I. Relación de planes estratégicos con un alcance temporal posterior a 2017 que han sido consultados y que están disponibles a través de las páginas web de las universidades públicas}

Universidad Autónoma de Madrid. Estrategia UAM 2025. http://www.estrategiauam2025.uam.es/pdf/UAM_Estrategia_2025.pdf [Consulta: 26/12/2017]

Universidad de Alcalá. Plan estratégico 2014-2018. http://www.transparencia. universidaddealcala.es/documentos/pdf/PLAN_ESTRATEGICO_UAH.pdf [Consulta: 26/12/2017]

Universidad de Alicante. UA40: plan estratégico 2014-2019. http://www.transparencia. universidaddealcala.es/documentos/pdf/PLAN_ESTRATEGICO_UAH.pdf [Consulta: 26/12/2017]

Universidad Carlos III de Madrid. Plan estratégico 2016-2022. http://hosting01.uc3m.es/ semanal3/documents/Plan_estrategico_2016_2022.pdf [Consulta: 26/12/2017]

Universidad Complutense de Madrid. Estrategia UCM2020 de Investigación. http://www.ucm.es/data/cont/docs/3-2015-12-10-PlanEstrategicoInvestigacionUCM.pdf [Consulta: 26/12/2017]

Universidad de Almería. Plan estratégico 2016-2019. http://cms.ual.es/idc/ groups/public/@varios/@planestrategico/documents/documento/plan_estrategico_docu mento.pdf [Consulta: 26/12/2017]

Universidad de Coruña. Plan estratéxico UDC2020. https://www.udc.es/export/sites/udc/ goberno/_galeria_down/veis/documentos/PLAN1320.pdf [Consulta: 26/12/2017]

Universidad de Extremadura. Plan estratégico 2014/2018. https://www.unex.es/archivos/ ficheros/PlanEstrategicoUEx.pdf [Consulta: 26/12/2017]

Universidad de Jaén. II Plan estratégico de la Universidad de Jaén: horizonte 2020. www10.ujaen.es/sites/default/files/users/planeuja/II\%20plan\%20 estrategico\%20UJA.pdf [Consulta: 26/12/2017] 
Universidad de la Laguna. Plan estratégico de investigación 2014-2020. https://www.ull.es/Private/folder/institucional/ull/wull/investigacion/novedades/plan_es trategico201420.pdf [Consulta: 26/12/2017]

Universidad de las Palmas de Gran Canaria. IV Plan Estratégico Institucional de la ULPGC (2015-2018). https://www.ulpgc.es/sites/default/files/ArchivosULPGC/noticia/ 2015/Dic/pei_20152018_d.pdf [Consulta: 26/12/2017]

Universidad de León. Líneas estratégicas 2017-2018. http://transparencia.unileon.es/ index.php/homepage/plan-estrategico [Consulta: 26/12/2017]

Universidad de Oviedo. Propuesta de plan estratégico para el periodo 2018/2022. http://www.uniovi.es/-/propuesta-de-plan-estrategico-para-el-periodo-2018-2022 [Consulta: 26/12/2017]

Universidad de Salamanca. Plan estratégico general 2013-2018. http://www0.usal.es/ webusal/files/PEG-USAL-APROBADO_ConsejoSocial.pdf [Consulta: 26/12/2017]

Universidad de Santiago de Compostela. Plan estratéxico 2011-2020. http://www.usc.es/export9/sites/webinstitucional/gl/servizos/calidade/descargas/Planific acion/planEstratexicoUSC.pdf [Consulta: 26/12/2017]

Universidad de Sevilla. Plan estratégico de la Universidad de Sevilla 2018-2025 (borrador). http://anticipa.us.es/sites/default/files/anticipa_borrador.pdf [Consulta: 26/12/2017]

Universidad de Valencia. Pla estratègic 2016-2019. https://www.uv.es/corporate/ peuv/PEUV_2016-19_v2.pdf [Consulta: 26/12/2017]

Universidad de Valladolid. Directrices Generales de Política Docente e Investigadora 2017-2018. http://planestrategicouva.es/wp-content/uploads/2017/11/Directrices-20172018-.pdf [Consulta: 26/12/2017]

Universidad Politécnica de Valencia. Plan estratégico 2015-2020. https://www.upv.es/noticias-upv/documentos/plan_estrategico_upv2020.pdf [Consulta: 26/12/2017]

Universidad Pompeu Fabra. Plan estratégico 2016-2025. https://www.upf.edu/web/plaestrategic [Consulta: 26/12/2017]

Universidad Rovira i Virgili. II Pla Estratègic de Recerca i Innovació de la Universitat Rovira i Virgili. http://www.urv.cat/media/upload/arxius/gabinet-comunicacio/plansestrategics/ii-pla-estrategic-recerca-innovacio-urv.pdf [Consulta: 26/12/2017]

\footnotetext{
AGRADECIMIENTOS

Al Ministerio de Educación, Cultura y Deporte (MECD) por el apoyo a la formación de jóvenes investigadores mediante el programa "Formación de profesorado universitario" (FPU), bajo el cual se realiza la presente investigación
} 